INTERNATIONAL DESIGN CONFERENCE - DESIGN 2018

https://doi.org/10.21278/idc.2018.0131

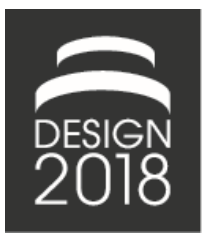

\title{
GOAL SETTING IN SUSTAINABLE BUILDING RENOVATION - EARLY PROTOTYPE DESIGN AND TESTING OF A NEW DECISION SUPPORT TOOL
}

\author{
A. N. Gade, R. L. Jensen, T. S. Larsen and S. B. Nissen
}

\begin{abstract}
This paper presents an early prototype of a new value-based decision support tool that can support building owners in setting goals for sustainability in renovation projects. The prototype includes the main functionalities of choosing and weighting criteria. Five users tested the prototype using think-aloud testing. The results showed that providing a pre-defined set of criteria for the goal setting made the users feel locked in their choices, and challenged in weighting criteria using the analytical hierarchy process. The results have informed the further design iterations of the prototype.
\end{abstract}

Keywords: decision making, conceptual design, prototyping, architectural design, design support system

\section{Introduction}

Multiple decisions have to be made throughout building renovation projects involving multiple decision makers. One of the early decisions professional building owners have to make is to set goals for the single renovation project or for multiple projects within a larger building portfolio. Research has shown that this goal setting is often implicit and is not done in a systematic way (Nielsen et al., 2017), even though this strategic area can be viewed as the rational heart of the entire process (Ferreira et al., 2013). Decision support tools are one solution to assist professional building owners during the early stages of renovation projects.

Various decision support tools for renovation already exist. In a review made by the authors, 43 decision support tools for renovation were found in the literature and analyzed with regard to where in the renovation they could support the decision makers (Nielsen et al., 2016). Nine of the tools included the aspect of setting goals in the pre-design phase of renovation projects. Two of these tools were value-based, in the sense that the renovation objectives were chosen based on the preferences of the decision makers and stakeholders. The rest of the tools were based on a fixed set of criteria but left the weighting of the criteria open to being assigned by the decision makers. One of the valuebased methods found in the review was RENO-EVALUE (Jensen and Maslesa, 2015), designed as a basis for dialogue among building professionals and building users. The other one is the Total Value Model (Blinkilde et al., 2011), which is a process tool aimed to support the building owner in setting strategic objectives for renovation projects. In addition to the tools found in the literature review, several new tools have emerged in the literature and the construction industry. Frame (BDB-metoden Aps, 2016) is a tool designed to support the building owner in managing the strategic vision from idea to construction and operation in both sustainable new buildings and renovation projects. A dialogue and prioritization tool based on gamification was developed by Hansen et al. to make complex issues 
of sustainability available to the residential democracy decision-makers in housing associations (Hansen et al., 2017). A value map framework was proposed by Kamari et al. to characterize essential areas to achieve a holistic building renovation in early design stages (Kamari et al., 2017). The tools mentioned above all support the decision makers in renovation projects, but none of these focus specifically on managing renovation activities within building portfolios. Furthermore, a majority of the existing tools found in the literature focus on the design process, whereas only a few emphasize the decisions made in the pre-design state.

This article presents the design process and testing of an early prototype of a new decision support tool that can support professional building owners in setting goals in renovation projects. The prototype provides a framework for choosing and weighting sustainability criteria. The prototype was designed using design methods from contextual design (Holtzblatt et al., 2005) and interaction design (Preece et al., 2015). Several design iterations were made internally in the research group before the concept was presented to potential users. Five potential users tested the interactive prototype through a Think-aloud test (Rubin and Chisnell, 2008) and were asked to give feedback on the prototype. The early testing was made to adjust the requirements and the design to the user's needs, to ensure usability and usefulness of the finished tool. The results of the tests were used to refine the requirements and future design iterations. The prototype presented in this paper consists of a goal setting module and is a part of a more comprehensive decision support tool aimed at professional building owners managing larger building portfolios. The decision support tool includes, in addition to setting goals, a module for registering the existing building and a cost estimation module, to support the building owner in prioritizing which buildings to renovate within a building portfolio.

The paper is structured as follows. First, the methods for the prototype design and testing is described. Second, the design process for the tool is elaborated including early sketches and prototype versions, followed by a presentation of the prototype that was tested in this paper. Third, the test results are presented. Lastly, the results are discussed and the conclusions summed up. This particular tool is necessary as existing decision support tools for building renovation, in the authors' opinion, do not support professional building owners sufficiently in setting goals for renovation projects in the predesign stage. The novelty of this approach lies in the focus on supporting building owners in choosing which buildings to renovate within a larger building portfolio through value-focused decision making (Keeney, 1992) in the pre-design stage.

\section{Methodology}

Methods from interaction design (Preece et al., 2015) and contextual design (Holtzblatt et al., 2005) such as affinity diagramming, scenarios, sketching, and user journey mapping have been used in the design and development of the prototype. The prototype was evaluated through think-aloud testing with five potential users. Think-aloud testing is a usability testing method where participants are asked to verbalize their thoughts while testing a system (Rubin and Chisnell, 2008). The advantages of using this type of testing is that is cheap and flexible, and therefore it is useful for getting early feedback from users. The goal of the testing process was that it should be open and explorative and that the users should say all that came to their mind while clicking through the prototype, regarding both the overall concept, functionalities, and design.

According to Nielsen (2000), the best usability test results come from testing with five users and running multiple smaller tests during the design process. Therefore, five potential users were invited to test the prototype at this stage. The test persons consisted of one building professional from a Danish municipality (a public building owner), one building professional with years of experience from an architectural company, and students in their final year of the professional bachelor degree educations Architectural Construction Technology Management and Energy Management. The test persons were asked to click through the interactive prototype on a PC, and verbalize their thoughts during the process. The prototype was interactive meaning that the buttons were active and the test persons could navigate between the different pages. However, it was not possible to enter text or tick of boxes, etc. Data were collected through note taking and audio recordings by the researchers taking a qualitative approach and were subsequently analyzed using grounded theory with open coding (Strauss and Corbin, 2008). The coding was done by first transcribing the audio recordings from the 
test sessions, and coding it in relevant themes that emerged from the data. The evaluation was made as a formative evaluation process, to get early feedback on the prototype, in order to inform the design process.

\section{Prototype development process}

This section describes the design and development process of the prototype, to provide insights into the rationale behind the design. The prototype version that was tested in this paper is presented in Section 3.1 .

The requirements for the new decision support tool have developed over time and have been informed by the design process and formative evaluations. The initial set of requirements were based on identified gaps in the literature (Nielsen et al., 2016). The initial requirements formed the base for empirical interviews with potential users, which has been documented in (Nielsen et al., 2017), to ensure that the practical needs of potential users were taken into account. The requirements included in this paper only concern the goal setting aspects of the tool.

\section{Requirements for the goal setting prototype}

Based in the preliminary literature review (Nielsen et al., 2016), it was found that only a few of the existing decision support tools for renovation could support the decision makers in setting goals in renovation projects, and even fewer were flexible with regard to the choice of sustainability criteria. To fill this gap, it was decided that the new tool should be value-based and encompass the preferences of the involved decision makers. Furthermore, a majority of the existing tools provided a fixed set of sustainability criteria, leaving no freedom of choice or flexibility to the decision makers to add their own, project-specific criteria.

Of the existing tools found in the literature, few of them were accessible, and the ones that were had not put much emphasis on the usability of the tools. Therefore, to ensure practical applicability of the tool, it is important that the tool is easy to use and have an intuitive user interface. This leads to the following tool requirements:

1. The tool should be value-based and encompass the preferences of the decision makers.

2. The tool should be flexible in the sense that the user can use project-specific criteria.

3. The tool should be easy to use and have an intuitive user interface.

Based on the interviews with professional building owners (Nielsen et al., 2017), which are intended to be the primary users of the tool, it was found that the professional building owners often did not have an explicit dialogue regarding the goals for renovation projects, both internally in the organisations or with advisors and stakeholders. This has led to the following requirements:

4. The tool should enhance dialogue among the decision makers in the pre-design state of renovation projects.

5. The tool should facilitate a group decision-making process.

Initially, affinity diagrams, several sketches and conceptual ideas for interface design and how users were intended to use and interact with the system was made. Scenarios were made at this early stage as a tool for communicating the concepts of the tool. Figure 1 shows a scenario sketch of how the decision makers can meet and set goals using the tool. The scenario starts with a building owner with the decision problem of which buildings to renovate within a building portfolio. Then, the building owner meets with advisors, decision makers within the organization, and relevant stakeholders to initially set goals for sustainability for the renovation projects, as a point of departure. The different priorities are then discussed. The decision support tool is used to structure the discussion process, and preliminary goals for the renovation projects are set. The sustainability goals are a balance between social, economic and environmental qualities, based on the definition of sustainability provided in the Brundtland Report (WCED, 1987). The primary users of the tool are intended to be professional building owners, e.g., municipalities, housing associations or real estate management companies, and the secondary users their advisors, such as engineers and architects. 

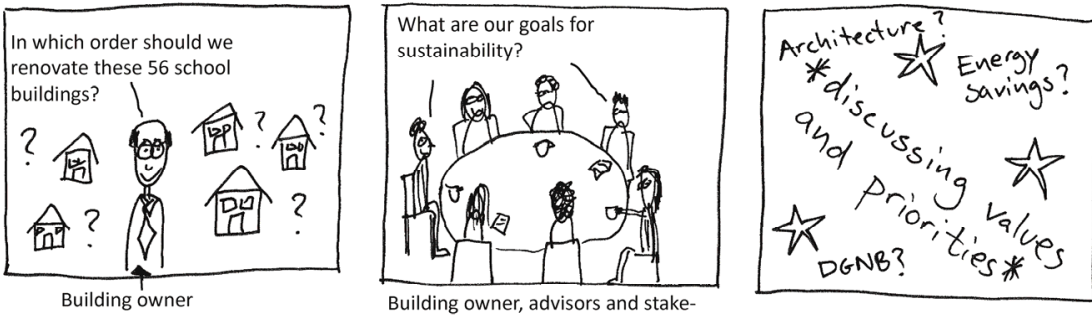

Building owner, advisors and stakeholders
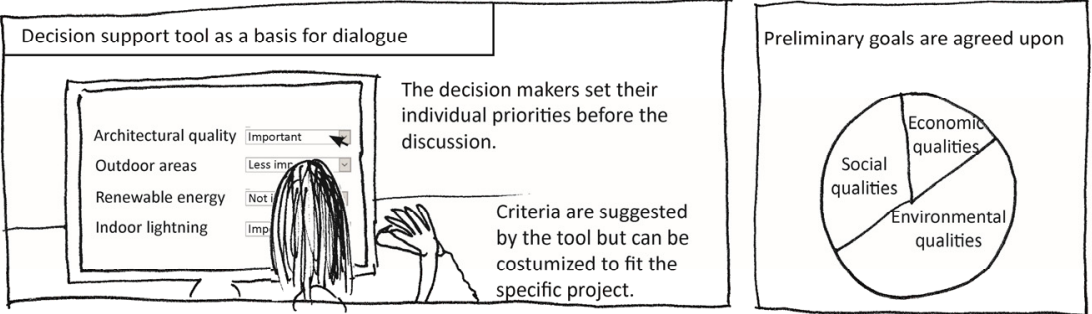

Figure 1. Sketch showing a scenario of how the decision makers can set goals

The first iteration of the prototype was made with hand-drawn sketches and conceptual drawings. The sketches were fragments of the idea and concept, including both process models and early interface ideas. The sketches were put into an interactive digital framework. This first interactive prototype was in the form of a mobile application (Figure 2). Figure 2 shows the initial screens when opening the application, including a login page and the main menu. The interface was hand-drawn, with the purpose of communicating the main functionalities but taking the focus on the detailed design of buttons, choice of colours, etc. The idea at that stage was that the system should be accessible from the phone because it is easy to carry along and potentially the users were carrying the phone with them anyway. This prototype was presented to one representative from the municipality of Aalborg, who gave feedback on these initial ideas, along with the initial scenario models. This feedback was documented through notes and was implemented in the next design iteration. Designing an interactive interface made it easier to communicate the ideas to users and other researchers.
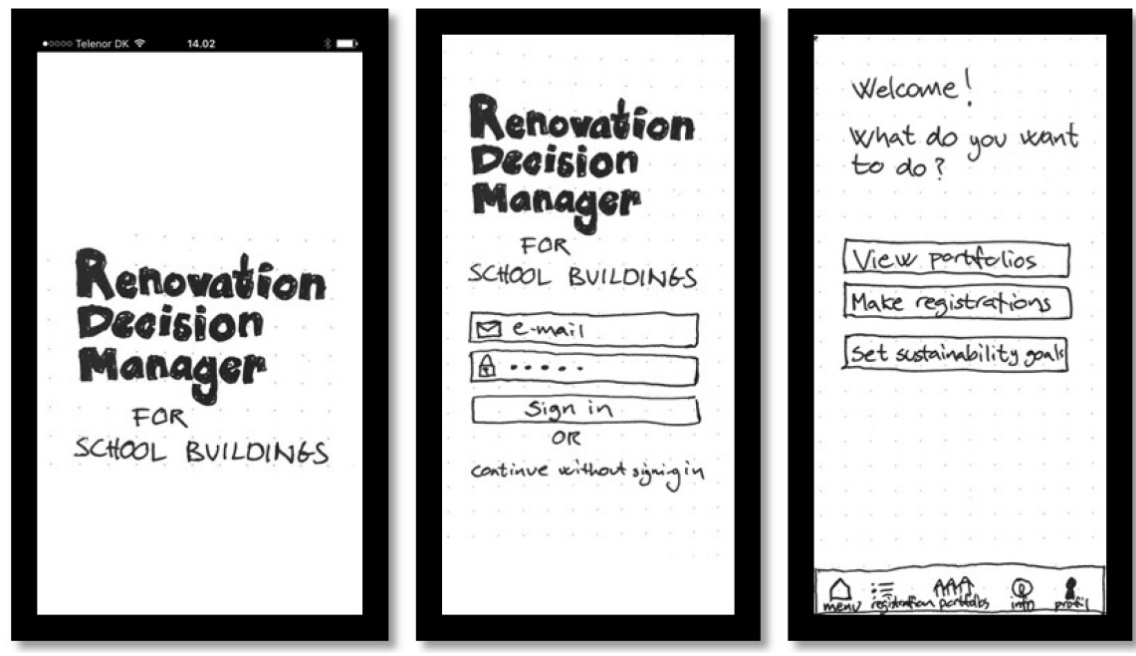

Figure 2. Sketches from the first prototype

The criteria used in the early prototypes were adapted from the sustainability assessment scheme DGNB-DK (Green Building Council Denmark, 2014) to provide a gross list to choose from. The analytic hierarchy process (AHP) (Saaty, 1990) was chosen as a method for weighting criteria, based on its successful implementation in several existing decision support tools for renovation and in the construction industry in general (Nielsen et al., 2016). 


\subsection{Prototype presentation}

The process of using the prototype that was tested in this study consists of two main steps. The first step is to choose sustainability criteria; this can be done either by using a set of pre-defined criteria from an assessment scheme such as BREEAM, LEED or DGNB, or by entering the building owner's own, project-specific criteria or a combination between the two. In the prototype, DGNB-DK (Green Building Council Denmark, 2014) criteria were used, with the option to add project specific criteria. Figure 3 shows the system architecture of the prototype. The two main steps are the choosing of sustainability criteria, and subsequently prioritizing these criteria. If project specific criteria are defined, it is important to note how these are measured and define the minimum and maximum acceptable levels for each criterion. After the criteria that reflect the goals of the building owners have been chosen, next step is to prioritize these criteria to determine their relative importance. The weighting process is based on AHP as in the earlier prototype iterations and uses pairwise comparisons of the criteria to express their relative importance. In the prototype, the main criteria groups are compared first, and afterward, the criteria in each criteria group are compared. The intention is that multiple decision makers can participate in weighting criteria, in this way multiple people from the building owner's organization, relevant advisors or stakeholders can be invited to participate in the weighting process. The group decision making aspects were not tested with this version of the prototype but are planned later in the research and development process. Instead, the tests that were made were from the individual decision maker's point of view.

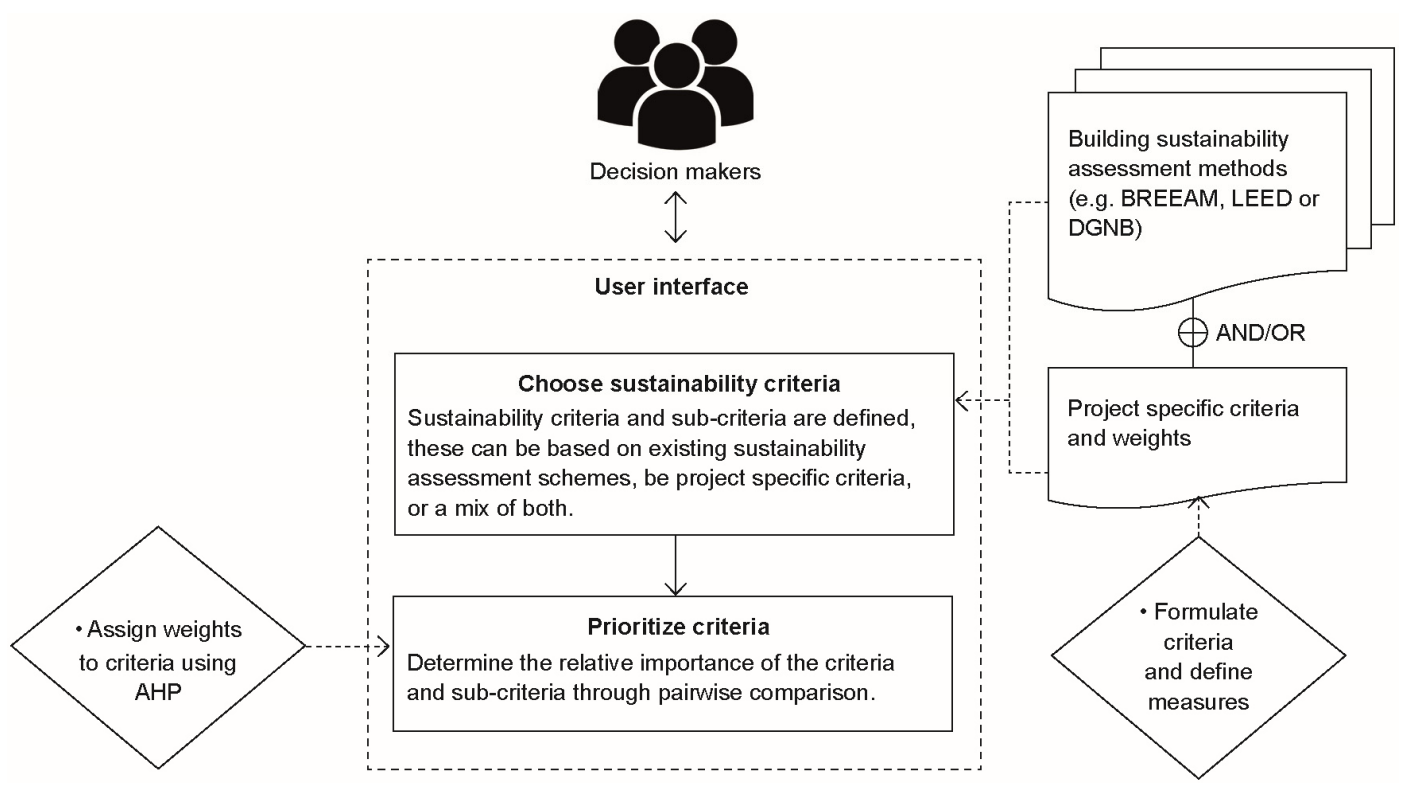

Figure 3. System architecture of the prototype

The interactive prototype was created using the Balsamiq software (Balsamiq Studios, 2017). The format of the prototype allowed the test persons to click through the prototype on a PC. The interface was designed as simple as possible in order to get the test persons to focus on the content, functionalities and the overall concept of the prototype, and not go into details with, e.g., colours, shape of buttons, etc., which will be saved for a later state and level of detail of the design. The user interface at this stage included a logo and name of the prototype in the top left corner, and a "back" and "forth" button on each page below the main text, written with letters and with an arrow indicating "back" or "forth." In addition to this simple navigation, the DGNBDK main criteria groups were placed on the right side of the screen with a small leaf next to each line (Figure 4), to give the user an overview of which criteria group was active on the main screen. Figure 4 shows a screenshot of an overview of the "social quality" criteria (from DGNB-DK). The user can tick the criteria on and off in the small box next to the criteria, indicating whether the criteria it is a goal to include the criteria in the project or not. At the end of the list is an empty box where the user can add additional criteria if the provided list is not sufficient to fit the project goals. In the right part of the screen is an interactive menu, making it easier for the user to navigate between the different categories of sustainability. 


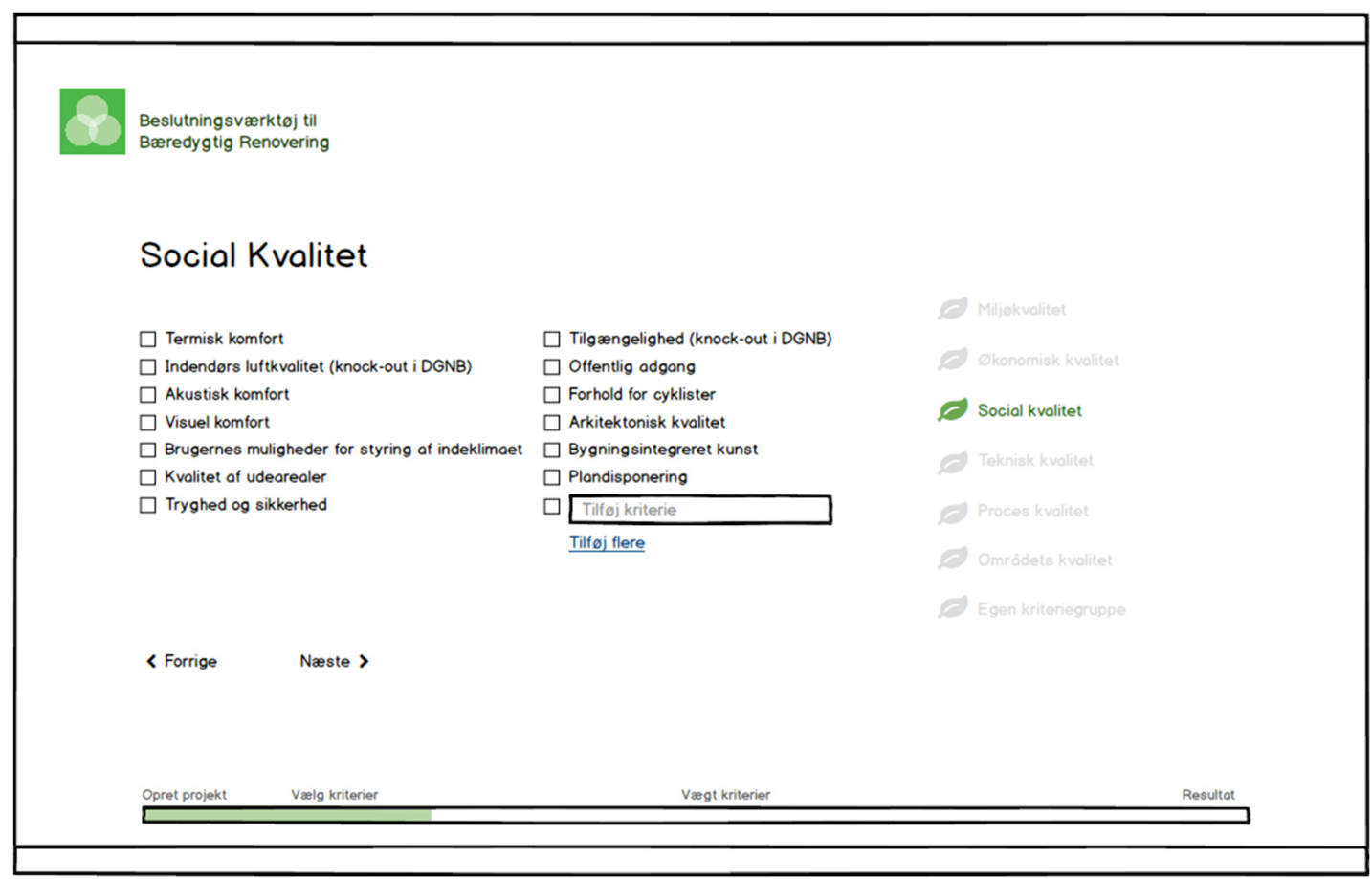

Figure 4. Screenshot showing the criteria for Social Quality

Figure 5 shows a screenshot of one of the criteria weighting screens, where the main criteria groups are prioritized against each other through pairwise comparison. In this example, the user has to mark one of the circles between the criteria, indicated whether the two criteria opposite each other are equally important, or how much more important one of the criteria is compared to the other according to the principles of AHP. The original AHP scale is a nine-point scale (Saaty, 1980), but for the sake of simplicity in the prototype only a five points were used as an example.

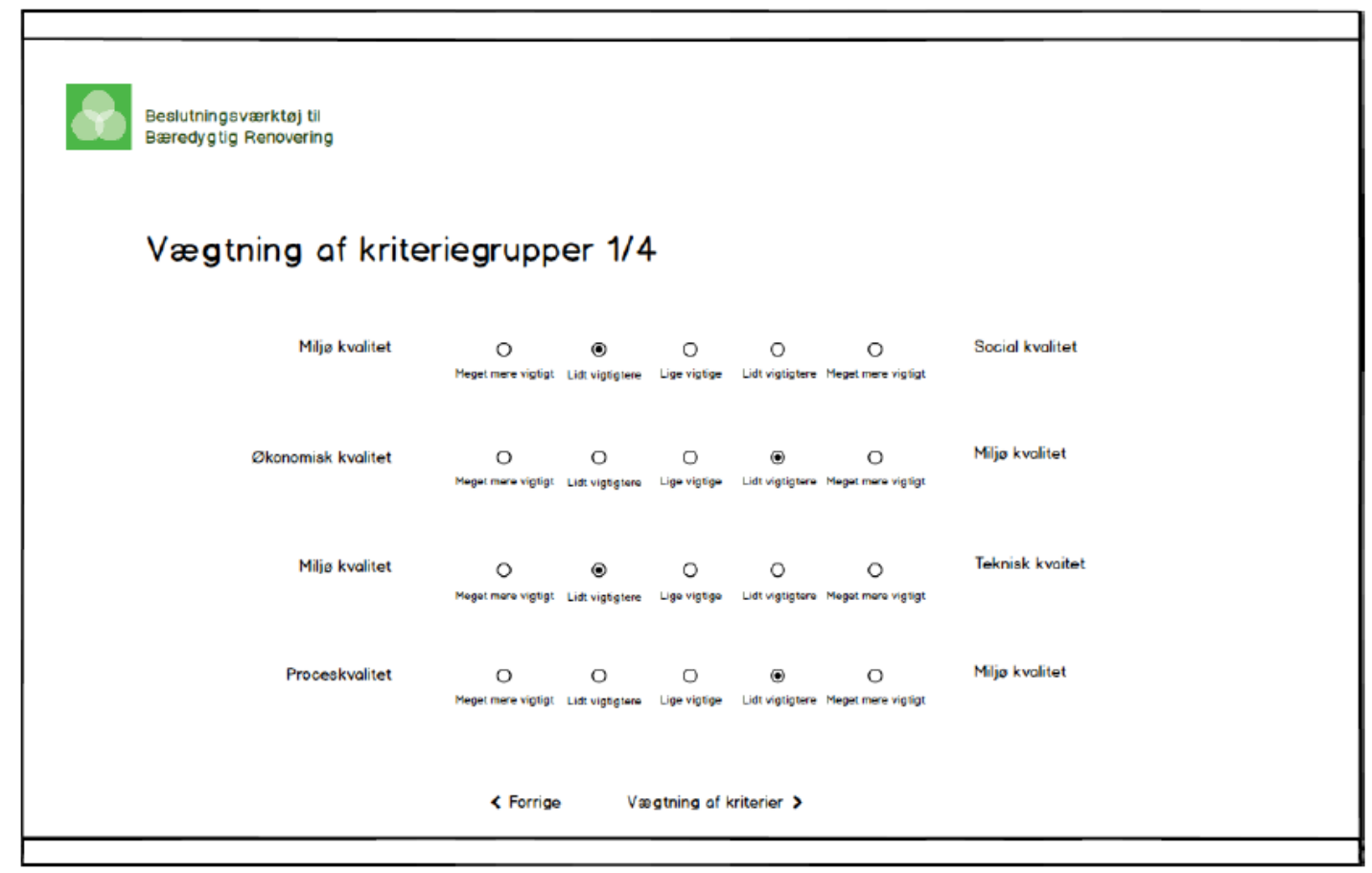

Figure 5. Screenshot showing the weighting of the criteria groups using AHP 


\section{Test results}

This section presents the results from the think-aloud tests from the five potential users. The data were analyzed using a grounded theory approach with open coding. All citations from the users were entered into a word document, printed out, and cut into small pieces of paper, each containing a citation. Then, the first author divided each statement into themes, by placing the physical pieces of papers in different piles. This was an iterative process, and the number of themes were reduced through each iteration, and, lastly, three main themes had emerged from the data. The results are presented according to the themes.

\subsection{User interface}

The users gave feedback on both usability and the concept in general. Regarding the user interface, the participants had detailed comments to specific details, even though the design was kept as simple as possible. It was expressed by the participants that a toolbar or an over-all navigation bar would make navigation easier to the user. It was suggested to create a small question mark next to the criteria for the user to click on to get a description of the respective criteria. Individual test persons suggested that it was important to mark the "knock out" DGNB-DK criteria and that the decision makers' own criteria should only be added to the "own criteria" page, and not be mixed with the DGNB-DK criteria. Also, it should be possible for the user to add notes on the "own criteria" page, and not in relation to the predefined DGNB-DK criteria. It was expressed that the user was presented with too much intro text and that this was okay the first time one should use the tool, but otherwise, it should be hidden on a separate page, e.g. "read more," that the user can click on if more background information is needed. Also, it was expressed that it was a bit confusing that the prototype gave the option of either set goals for a single building or several buildings, and that it should focus on either one or the other.

\subsection{Choice of criteria}

Regarding the choice of criteria, it was expressed by several test persons that it is important that the user understands that it is only if sustainability assessment, using e.g. the DGNB-DK assessment scheme, not is the end goal that the criteria should be weighted. If assessment is a goal, the DGNB-DK manual should be followed, including the weights in the assessment scheme. It is suggested that a diagram is presented to the user to show the weights of the DGNB-DK scheme compared to the weights that are generated through the prototype by the user. Also, it is suggested that it is illustrated how the different criteria affect each other. This could be predefined if a set of known criteria are used, but if the user enters own criteria, it becomes more challenging and would require the user to provide sufficient information about each criterion for the system to be able to recognize which other criteria will be affected. It was expressed that choosing between and weighting the DGNB-DK criteria requires a high level of knowledge about DGNB-DK from the individual user. It is therefore important to include additional information and go into details about each criterion. Also, it should be made clear to the user that the criteria presented are in addition to the mandatory building regulations, and are an extra step towards sustainable solutions. Furthermore, it should be made more clear that the user should only add own criteria if the goal is not to certify the buildings using DGNB. Several test persons expressed that it was important to give the user information on why the DGNB-DK criteria were used and that the user can choose early if assessment is a goal or not. It can seem confusing to the user that the DGNB-DK criteria are used, but not the DGNB-DK weights, and that the user has to weigh the criteria themselves. One test person expressed that the prototype encourages the users to use DGNB-DK, so it was hard to see why one should add own criteria. If it is a part of the concept that the user should add own criteria, this should be explained better. Also, it was addressed that it could be "dangerous" that the user can leave out too many of the DGNB-DK criteria, as the project would possibly no longer be sustainable in relation to the DGNB-DK definition.

The test persons suggested that the user gets feedback on whether the chosen criteria could be enough to achieve DGNB silver, gold or platinum. However, at this stage of the process, the level of information is not sufficient to give this kind of estimate, as it would require exhaustive documentation on the building design in relation to each criterion. This tool is intended as a part of the early design process, where the direction of the project is set, and not to be used in the detailed design phase where 
documentation for e.g., DGNB-DK assessment is produced. Another suggestion was to give the users feedback on how sustainable the combination of criteria chosen are in comparison to, e.g., DGNB. This is an interesting idea and is possible as long as the criteria groups are comparable. It is difficult if the user enters many project-specific criteria, as these do not necessarily compare to, e.g., the DGNB framework. If only criteria from DGNB-DK is chosen, it would be possible to give the user visual feedback on the "level of sustainability," meaning the balance between social, environmental, economic aspects. It was also requested that an illustration of the process the user has to go through using the tool be presented on the intro page.

\subsubsection{Data}

It was suggested to store the project-specific criteria from project to project, to be used in future projects. Along the same line, it was expressed that a "save" function is important to integrate, to make it possible to go back and see earlier prioritizations and projects. In that way, the users can also see if the goals have changed over time. Also, it was expressed that benchmarking between similar user groups could be useful, e.g., among different municipalities. In that way, it is possible to compare similar projects. Another idea suggested by one of the test persons was to work closely with the users to define project specific criteria and integrate these in the tool from the beginning.

\subsection{Criteria weighting}

Regarding the weighting of the criteria, it was suggested that numbers are put below the circles (that are used to mark the prioritization during the pairwise comparisons of criteria) and that it should be possible to add notes in relation to each pairwise comparison, to document the rationale behind the prioritizations. A visual representation of the criteria weights was suggested to be shown after all criteria are weighted. Several of the test persons expressed that it was hard to weigh the criteria groups, as it was hard to understand the consequences. This might be easier when the user has a specific project in mind otherwise, it might be an abstract exercise. Also, it was expressed by several test persons that it was difficult to prioritize the sub-criteria because they were all basically were perceived as important. During the criteria weighting, it was expressed by several test persons that more than just the criteria name was needed to fully understand the meaning and the extent of the individual criteria. Also, it was expressed that the order of the criteria during the weighting process should be explained to the user. This aligns with the earlier expression that the overall process should be presented to the user in the introduction page.

One test person expressed the idea that the choosing of criteria and the weighting process could be integrated and done in one process instead of two, e.g. when a criterion is chosen, the user could express a 1-3 or 1-5 prioritization directly in relation to the criteria. The same person suggested that it would be easier to make a rough prioritization at an early state, e.g., "low," "medium" or "high" priority, to have a point of departure, and then make a more detailed weighting at a later state were more information about the project were accessible. Overall, it was expressed that the AHP weighting method was "heavy," time-consuming and too comprehensive. One test person expressed that it was very difficult to prioritize the criteria on an overall, strategic level, or at a very early state having a specific building in mind. The level of detail of the criteria would be useful later, e.g., when a specific building brief had to be created, it was expressed.

\section{Discussion and conclusions}

This paper presents the development and content of a tool developed to support professional building owners in setting goals for renovation projects. An early prototype was tested with five potential users using think-aloud tests to get early feedback on the prototype regarding the overall concept, including functionalities and design. The testing was explorative, in order to gain useful feedback to inform the future design process. The rest results were analyzed using a grounded theory approach, and the data were coded using open coding. The overall themes that emerged from the data were "user interface," "choice of criteria" and "criteria weighting." Regarding the user interface, the test persons gave feedback on the design, even though it was kept simple due to the early design stage. The design feedback has been 
evaluated and used in the design of the forthcoming prototype iterations. In the prototype, sustainability criteria from DGNB-DK were used as a point of departure for choosing criteria, with the option to add the users' own project-specific criteria. Providing the DGNB-DK criteria as a starting point made the test persons feel "locked", and it was hard for them see the meaning of even adding project-specific criteria, as DGNB-DK in their opinion was sufficient and covered the relevant criteria. The test persons did not have a specific building or buildings in mind during the test, and that might affect this perception. It might be easier to choose criteria having a specific project in mind, in order to see if any project specific criteria were needed in the specific case. Based on the results, it can be concluded that if the tool is to be flexible with regard to choosing criteria, less emphasis should be put on the DGNB-DK criteria, or they should not necessarily be introduced as a part of the tool. On the other hand, it is useful to provide some sort of gross list of criteria, but these could be made without a direct connection to a sustainability assessment scheme. Along the same lines, it confused the test persons why they should even weight the criteria, instead of just using the criteria weights inherent in DGNB-DK. The idea behind weighting the criteria is that the tool should be flexible, and can be used even though the aim is not to assess the finished building(s) using a sustainability assessment scheme. Regarding the weighting of criteria, the participants found it hard, nonintuitive and un-transparent to use AHP. One reason for this might be because the users did not have a specific project in mind during the test, and that the weighting of criteria was therefore too abstract. Still, the test results lead to the conclusion to challenge the use of AHP and to introduce a less complex alternative weighting method in future prototype iterations.

It should be recognized that in practice, the objectives of the decision makers are often developed throughout the design process, and the values might therefore not be clear until the design alternatives have been generated (Wang and Zeng, 2010). However, we argue that having a dialogue among the main decision-makers, and potentially stakeholders, at the beginning of a renovation project can provide a clear direction for the project, even though the criteria and their importance might change during the design process, when more information about the project is available, and the decision foundation is stronger. In relation to existing decision support tools for building renovation, a majority of the tools that include the aspect of goal setting provide an initial set of criteria for the decision makers to choose from. RENOEVALUE provides four criteria categories for renovation, including eight parameters and 38 factors (Jensen and Maslesa, 2015). The tool presented in this paper used the 40 sustainability criteria, divided into five main categories, from DGNB-DK. Though the criteria differ in the two tools, the main themes or categories overlap. However, the tool presented in this paper provides the option of adding the decision makers' own criteria to the criteria set and thereby providing a flexible framework for the building owner. The Total Value model provides a set of overall themes related to the different stages of renovation projects, including relevant questions for the building owners of important aspects that should be considered (Blinkilde et al., 2011). The open questions are designed to guide the building owner and make sure that relevant aspects are considered throughout the different phases of the project. In that way, the Total Value model is value-based, but does not suggest specific criteria for the decision makers to choose from, and does therefore not directly support the decision makers in setting explicit goals, as the new decision support tool presented in this article does. The gamification dialogue tool presented by Hansen et al. applies the criteria from DGNB-DK, however, the criteria are transcribed into layman terms to make the tool accessible to non-building professionals. The main purpose of the dialogue tool is to improve the dialogue of sustainability among building professionals and non-building professionals such as occupants. This approach could be an interesting extension to the tool presented in this paper, and could potentially broaden the use of the tool to support the communication between the building owner and occupants or other stakeholders who are not building experts. The Value Map (Kamari et al., 2017) was designed to characterize essential areas to achieve a holistic building renovation in early design stages. However, the Value Map does not directly support the decision maker in the decision-making process, but provides a "map" that can be used for discussing priorities. The Value Map could, therefore, be a valuable addition to the goal setting tool presented in this paper, to make the tool more independent of the sustainability assessment methods. However, as the goal might be to assess the buildings after renovation, the option of applying the criteria and weights from the assessment methods should still be an option as a part of the tool. 
The results presented in this paper will be used to inform the ongoing design process of a new decision support tool designed to support the professional building owner in choosing which buildings to renovate within a building portfolio, including the aspect of setting goals for sustainability, which was the main focus of this paper. Furthermore, the results can support both researchers and building professionals in designing new decision support tools for sustainable renovation.

\section{Acknowledgements}

The authors kindly thank the test persons for taking the time to evaluate the prototype.

\section{References}

Balsamiq Studios (2017), Balsamiq software. [online] Balsamiq. Available at: https://balsamiq.com/

BDB-metoden Aps (2016), BDB-metoden. [online] Available at: http://bdb-metoden.dk/ (accessed 09.05.2016).

Blinkilde, H., Jensen, N.-A., Kongebro, S., Petersen, P.T., Rasmussen, J. et al. (2011), Totalvardimodellen, Plan $\mathrm{C}$, Albertslund, Denmark.

Ferreira, J., Pinheiro, M.D. and de Brito, J. (2013), "Refurbishment decision support tools review-Energy and life cycle as key aspects to sustainable refurbishment projects", Energy Policy, Vol. 62, pp. 1453-1460. https://doi.org/10.1016/j.enpol.2013.06.082

Green Building Council Denmark (2014), DGNB System Denmark 1.1., DK-GBC.

Hansen, H.T.R., Knudstrup, M. and Pedersen, S.S. (2017), "Gamification as a Means to User Involvement in Decision-Making Processes for Sustainable Buildings", Proceedings of WSBE17 / the World Sustainable Built Environment Conference 2017, Hong Kong, June 5-7, 2017, pp. 2531-2536.

Holtzblatt, K., Wendell, J. and Wood, S. (2005), Rapid Contextual Design: A How-to Guide to Key Techniques for User-Centered Design (Interactive Technologies), Morgan Kaufmann.

Jensen, P.A. and Maslesa, E. (2015), "Value based building renovation - A tool for decision-making and evaluation", Building and Environment, Vol. 92, pp. 1-9. https://doi.org/10.1016/j.buildenv.2015.04.008

Kamari, A., Corrao, R. and Kirkegaard, P.H. (2017), "Sustainability focused decision-making in building renovation", International Journal of Sustainable Built Environment, Vol. 6 No. 2, pp. 330-350. https://doi.org/10.1016/j.ijsbe.2017.05.001

Keeney, R.L. (1992), Value-Focused Thinking : A Path to Creative Decisionmaking, Harvard University Press, Cambridge, MA, USA.

Nielsen, A.N., Jense, R.L., Larsen, T.S. and Nissen, S.B. (2016), "Early stage decision support for sustainable building renovation - A review", Building and Environment, Vol. 103, pp. 165-181. https://doi.org/10.1016/j.buildenv.2016.04.009

Nielsen, A.N., Larsen, T.S., Jensen, R.J. and Nissen, S.B. (2017), "Decision-making in the Pre-design Stage of Sustainable Building Renovation Projects", Proceedings of WSBE17 / the World Sustainable Built Environment Conference 2017, Hong Kong, June 5-7, 2017, pp. 1967-1972.

Nielsen, J. (2000), Why You Only Need to Test with 5 Users. [online] Nielsen Norman Group. Available at: https://www.nngroup.com/articles/why-you-only-need-to-test-with-5-users/ (accessed 21.11.2017).

Preece, J., Sharp, H. and Rogers, Y. (2015), Interaction Design - Beyond Human-Computer Interaction, John Wiley \& Sons Ltd., Chichester, UK.

Rubin, J. and Chisnell, D. (2008), Handbook of usability testing: How to plan, design, and conduct effective tests, 2nd ed., Wiley Publishing Inc., Indianapolis, Indiana.

Saaty, T.L. (1980), The Analytic Hierarchy Process, McGraw-Hill.

Saaty, T.L. (1990), "How to make a decision: The analytic hierarchy process", European Journal of Operational Research, Vol. 48 No. 1, pp. 9-26. https://doi.org/10.1016/0377-2217(90)90057-I

Strauss, A. and Corbin, J. (2008), Basics of qualitative research: Grounded theory procedures and techniques, SAGE Publications.

Wang, H.J. and Zeng, Z.T. (2010), “A multi-objective decision-making process for reuse selection of historic buildings", Expert Systems with Applications, Vol. 37 No. 2, pp. 1241-1249. https://doi.org/10.1016/j.eswa.2009.06.034

WCED (1987), Report of the World Commission on Environment and Development: Our Common Future (The Brundtland Report), Oxford University Press, Oxford.

Anne Nørkjær Gade, PhD student

University College of Northern Denmark, Energy and Environment

Sofiendalsvej 60, 9200 Aalborg SV, Denmark

Email: anni@ucn.dk 